This is a postprint version of the following published document:

Terrones, M., Martín, O., González, M., Pozuelo, J., Serrano, B., Cabanelas, J. C., Vega-Díaz, S. M. y Baselga, J. (2011): Interphases in Graphene Polymerbased Nanocomposites: Achievements and Challenges. Advanced Materials, 44 (23), pp.: 5302-5310.

DOI: $10.1002 / \mathrm{adma} .201102036$

(C) WILEY-VCH, 2011 


\title{
Interphases in Graphene Polymer-based Nanocomposites: Achievements and Challenges
}

\author{
Mauricio Terrones, Olga Martín, María González, Javier Pozuelo, Berna Serrano,Juan C. Cabanelas, \\ Sofía M. Vega-Díaz, and Juan Baselga*
}

Graphene constitutes a two dimensional sp ${ }^{2}$ hybridized carbon material with outstanding electrical and mechanical properties. To date, novel methods for producing large quantities of graphene and its derivatives (doped or function-alized graphenes, nanoribbons and nanoplatelets) are emerging, and research dedicated to the fabrication of polymer nanocomposites using graphenes has started. In this Research News, we summarize the synthesis and properties of graphene and its derivatives, and provide an overview of the latest research dedicated to the fabrication of polymer composites for different applications, including mechanical, electrical, optical and thermal. Some of the recently fabricated composites exhibit outstanding properties, however, it is vital to understand the chemistry and physics of the interphases established between the polymer and the graphene surfaces. The challenges in the fabrication of super robust and highly conducting composites using graphenes are also discussed. It is believed that graphene-based polymer composites will result in commercial products if their interphases and reactivity are carefully controlled.

\section{Introduction}

\subsection{Structure and Physico-Chemical Properties of Graphene}

Graphene consists of a single layer of $\mathrm{sp}^{2}$ hybridized carbon atoms forming a two-dimensional (2D) hexagonal lattice (Figure 1). It could be considered as a fundamental building block for all sp ${ }^{2}$-hybridized carbon allo-tropes (e.g. graphite, nanotubes and fullerenes). Possibly the fi rst experimental report on graphene appeared, ${ }^{[1]}$ in 1962. However, it was until 2004, when the Manchester group lead by A. Geim and K. Novoselov, [2] isolated individual graphene layers via repeated peeling using "scotch tape". In particular, graphene is a unique monolayer 2D crystal displaying a room temperature quantum Hall effect, [3] and more importantly, it is the strongest material reported to date. ${ }^{[4]}$ It is a semimetal with its conduction band and valence

band being degenerate at the $\mathrm{K}$ point in the Brillouin zone, and it has an extremely high room temperature carrier mobility (ca. 2 orders of magnitude greater than that of silicon). ${ }^{[5]}$

It is important to emphasize that as the number of graphene layers starts to pile up, the properties of the material starts to approximate that of graphite (a 3D crystal consisting of stacked layers of $\mathrm{sp}^{2}$ hybrid-ized carbon atoms; Figure 1). For example, bi-layer graphene exhibits parabolic electronic bands and the energy gap on bi-layer graphene could be opened, which is of interest in techno-logical applications. ${ }^{[6]}$ Tri-layer graphene displays a band struc-ture which combines the mono-layer and the bi-layer. As the number of layers increases, the conduction and valence bands start overlapping. ${ }^{[2,7]}$ Therefore, from the electronic standpoint, it is important to distinguish between the different types of graphenes: graphene, few-layer graphene, and graphite (Figure 1).

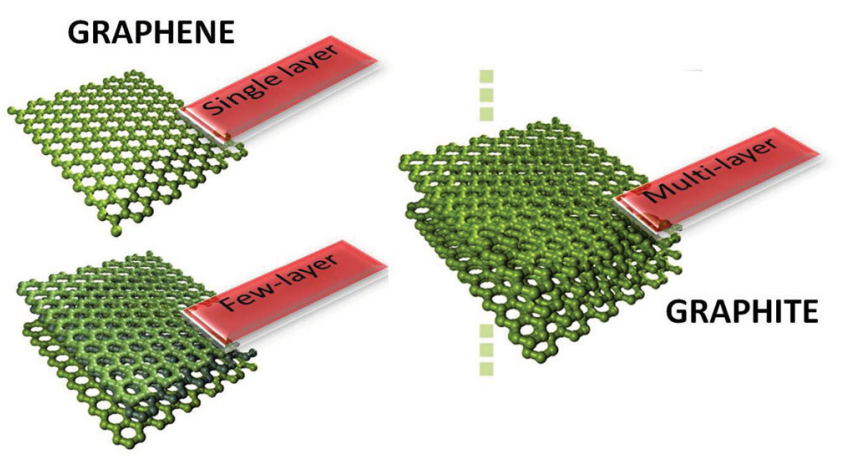

Figure 1. Molecular models of single- and few-layer graphene (left), and multi-layer graphite (right).

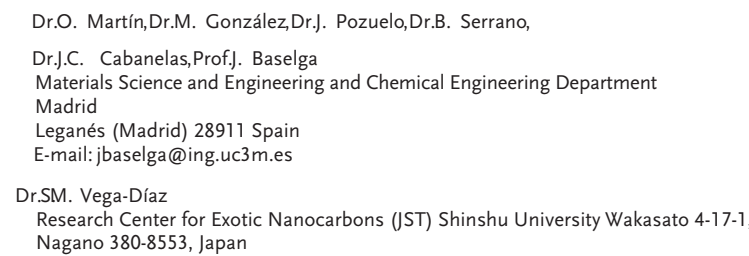

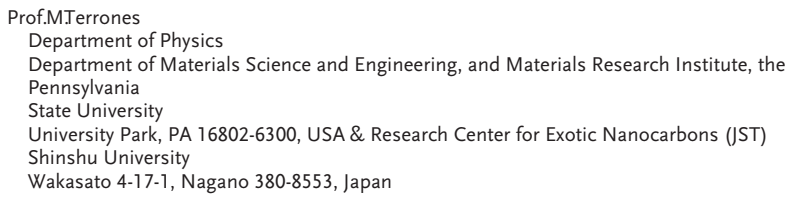


Interestingly, the stacking order of the layers has been found to dramatically change the electronic properties of multi-layer graphene.

The thermal properties of suspended graphene have exhibited extremely high values of thermal conductivity (4.84 \pm $0.44) \times 10^{3}$ to $(5.30 \pm 0.48) \times 10^{3} \mathrm{~W} \cdot \mathrm{m}^{-1} \cdot \mathrm{K}^{-1}$, higher than experimental values obtained for carbon nanotubes and diamond. ${ }^{[8]}$ More recent experimental measurements on chemical vapor deposition (CVD) grown graphene revealed lower values $\left(\right.$ ca. $\left.2500 \mathrm{~W} \cdot \mathrm{m}^{-1} \cdot \mathrm{K}^{-1}\right)$. ${ }^{[9]}$ These results indicate that the crystallinity of the $2 \mathrm{D}$ sheets affects significantly the thermal properties. Therefore, if one is to exploit the outstanding thermal properties of graphene, it is important to synthesize extremely high crystalline graphene so that the fabrication of heat dissipaters and polymer composites with high thermal conduction could be possible. In addition, the thermal properties of dopedgraphenes and individual graphene nanoribbons still need to be explored, as these novel forms of graphene could also have relevance in thermal applications.

Regarding the mechanical strength of an individual graphene sheet, graphene breaking strengths are 200 times larger than that of steel, and Young's modulus is ca. 1 Tera Pascal (TPa). ${ }^{[4]}$ Similar to the thermal properties explained above, these measurements were dependent on the number and types of defects within the 2D sheet. It is noteworthy that the predicted Young's modulus for graphite along the basal plane is ca. $1 \mathrm{TPa}$, and other mechanical measurements on individual graphene sheets have revealed Young's modulus values of ca. $0.5 \mathrm{TPa} .{ }^{[10]}$

The relationship between mechanical and electronic properties of graphene has been explored recently using CVD grown graphene and subjected to mechanical strains. ${ }^{[11]}$ When graphene was bent, the resistance (initially ca. $300 \Omega$ ) increases almost one order of magnitude. Similarly, the resistance could vary when stretching the sheet, and one order of magnitude difference between the resistance parallel and perpendicular to the stretching direction was found.

From the chemical standpoint, it has been demonstrated that highly crystalline graphene surfaces appear to be chemically inert, and these surfaces could interact with other molecules via physisorption ( $\pi-\pi$ interactions). However, the edges of graphene and graphene nanoribbons are more chemically reactive (Figure 2) and could indeed anchor different chemical groups including carboxyl $(\mathrm{COOH})$, carbonyl $(\mathrm{COH})$, hydrogenated $(\mathrm{CH})$ and amines $\left(\mathrm{NH}_{2}\right)$. The chemical activity could change dramatically at these edges, depending on their carbon termination (e.g. armchair or zigzag). One way to make the graphene basal surface more chemically reactive could be achieved by introducing surface defects (Figure 2) or high degrees of curvature.

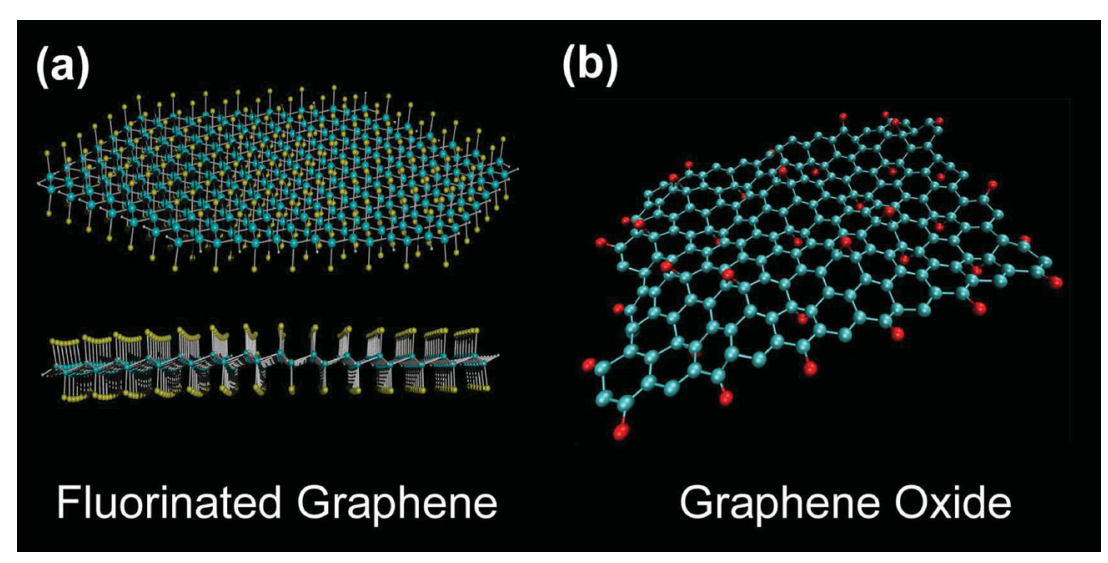

Figure 3. Molecular models of: a) single-layer fluorinated graphene (courtesy of T. Hayashi) and b) single-layer oxidized graphene (courtesy of R. Cruz-Silva and F. Tristán-López).
An alternative route to make the graphene more reactive consists of adding fluorine. This process (called fluorination) has been known for decades. However, only recently graphene has been fluorinated using different techniques including plasma treatments and $\mathrm{F}_{2}$ high temperature treatments (Figure 3). ${ }^{[12,13]}$ From the materials point of view, fluorinated graphene could be homogenously dispersed in different solvents to prepare polymer composites and further experiments are needed along this direction. It is also important to note that the electronic properties of graphene could be tuned via controlling the degree of fluorination, which modulates the energy band gap of the material. ${ }^{14]}$

Sofo and co-workers ${ }^{[15]}$ predicted the possibility of reacting the graphene surface with hydrogen atoms; the sheet was termed "graphane", and it was reported experimentally by Novoselov. ${ }^{[16]}$ However, the synthesis of this doped graphene still needs to be 
enhanced, and alternative types of analyses, related to the nature of the $\mathrm{C}-\mathrm{H}$ bonding using X-ray photoelectron spectroscopy (XPS), need to be carried out. In the case of graphene nanoribbons, fluorination and hydrogenation reactions remain to be carried out, as well as XPS and other allied studies.

Another way to fully functionalize the graphene surface is by oxygenation. The resulting material is known as graphene oxide (GO), and it contains different $\mathrm{C}-\mathrm{O}$ functional groups (e.g. epoxy, carbonyl and carboxyl groups). These materials are remarkably hydrophilic and more reactive. ${ }^{[17]} \mathrm{GO}$ is usually synthesized via chemical routes, and the material could also be dispersed easily in different solvents for generating robust polymer composites. ${ }^{[18]}$ These sheets contain a large number of defects, and further details about their preparation and applications could be found elsewhere. ${ }^{[18]}$

\subsection{Synthesis of Graphene and Graphene Nanoribbons}

\subsubsection{Graphene}

Besides the "scotch tape" technique used to obtain graphene layers, researchers have developed alternative routes to graphene. The first one describes the growth of ultrathin epitaxial graphite films by thermal decomposition on the (0001) surface of hexagonal SiC. ${ }^{[19]}$ A second method describes the synthesis of single- and few-layer graphene using an ambient pressure CVD method on polycrystalline Ni substrate. ${ }^{[20]}$ This method was subsequently modified using different carbon sources (polymer films and small molecules deposited on a metal catalysis substrate). ${ }^{[21]}$ Large area graphene sheets have also been synthesized by Ruoff via a CVD approach involving $\mathrm{CH}_{4}$ and $\mathrm{H}_{2}$ mixtures at $1000{ }^{\circ} \mathrm{C}$ on $\mathrm{Cu}$ foils. ${ }^{[22]}$ Unfortunately, the quantity of graphene produced using these routes is very low for polymer composite applications. Therefore, alternative routes to produce large quantities of graphene, graphene nanoribbons or graphene platelets need to be explored.

\subsubsection{Graphene Nanoribbons}

CVD is now able to synthesize large quantities of graphene nanoribbons (GNRs) via the thermal decomposition of ferrocene:ethanol:thiophene at $950{ }^{\circ} \mathrm{C}$ (Figure 4). ${ }^{[23]}$ However the formation of graphite nanoribbons by the decomposition of $\mathrm{CO} / \mathrm{H}_{2} / \mathrm{Fe}(\mathrm{CO})_{5}$ at $400-700{ }^{\circ} \mathrm{C}$ was reported in $1990 .{ }^{[24]}$ Alternative chemical routes, consisting of the exfoliation of commercial graphite, ${ }^{[25]}$ or by organic chemistry approaches, have also been recently developed (Figure 4). Templates have also been used during CVD in order to obtain GNRs. ${ }^{[26]}$ Here, the authors used the decomposition of $\mathrm{CH}_{4}$ on $\mathrm{ZnS}$ ribbons (used as templates) followed by acid treatments in order to remove $\mathrm{ZnS}$ (Figure 4). From a pure chemical approach, short GNRs have been synthesized by linking tetra- and hexa-phenylbenzenes via the Suzuki-Miyaura reaction. ${ }^{[27]}$ The produced ribbons are crystalline and exhibit lengths of 8-12 nm, although the quantities may not be very high. Hydrothermal processes have also been used to obtain either amorphous or crystalline carbon ribbons involving Teflon-lined autoclaves. ${ }^{[28,29]}$ Other approaches to nanoribbons use electrophoretic deposition on highly oriented pyrolytic graphite (HOPG) followed by a heat treatment. ${ }^{[30]}$
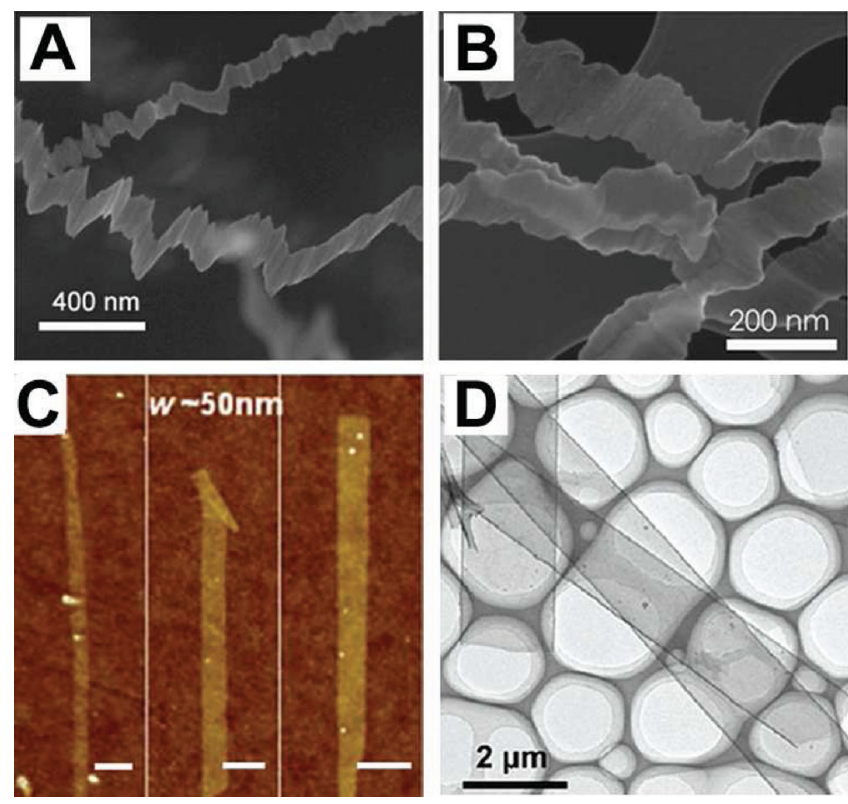

Figure 4. (a) and (b) SEM images of graphitic nanoribbons produced by CVD; ${ }^{[23]}$ (c) AFM image of chemically derived nanoribbons from graphite (scale bars $=100 \mathrm{~nm}$ ), ${ }^{[25]}$ and (d) low magnification TEM image of the nanoribbons produced by the ZnS template method. ${ }^{[26]}$ (Adapted with permissions from American Chemical Society and American Association for the Advancement of Science).

One way to generate large amounts of GNRs or nanoplatelets (GNPs) consists of unzipping multi-walled carbon nanotubes (MWCNTs). This method is advantageous when compared to the methods described above because: 1) MWNTs are currently produced in ton quantities by various companies worldwide and therefore, massive amounts of GNRs or GNPs could now be generated, and 2) The fact that GNRs and GNPs have plenty of edge surface makes these materials attractive for the covalent functionalization of polymers, and therefore the polymer composite fabrication. The first methods developed to obtain graphitic nanoribbons from nanotubes were published in 2009 and 2010. ${ }^{[31-36]}$ A comprehensive review on the properties and synthesis of GNRs has been reported elsewhere. ${ }^{[37]}$ Jia et al. ${ }^{[38]}$ noted that when using Joule heating and high electron irradiation, the most stable edges in graphene ribbons are either zigzag or armchair edges. At present, it is important to device GNRs and GNPs exhibiting sharp and crystalline edges so that specific polymer reactions could be controlled.

\subsubsection{Doped Graphene Nanoribbons}

Doping of graphenes, GNRs and GNPs is important because the electronic and quantum transport properties could be significantly modified. These properties depend on the nature of the dopants, their location and their concentration. Schematic representations of the substitutional and pyridine-like nitrogen doping in graphene nanoribbons are shown in Figure 5a-b. Theoretical studies on graphene nanoribbons doped with $\mathrm{N}, \mathrm{B}$, and $\mathrm{O}$ atoms have been investigated by Cervantes-Sodi. ${ }^{\left[{ }^{[9]} \text { How- }\right.}$ ever, doping of GNRs and GNPs could also be achieved using B, $\mathrm{P}$ or $\mathrm{S}$ (Figure $5 \mathrm{c}-\mathrm{d}$ ). In GNRs, the edge-type and substitutional 

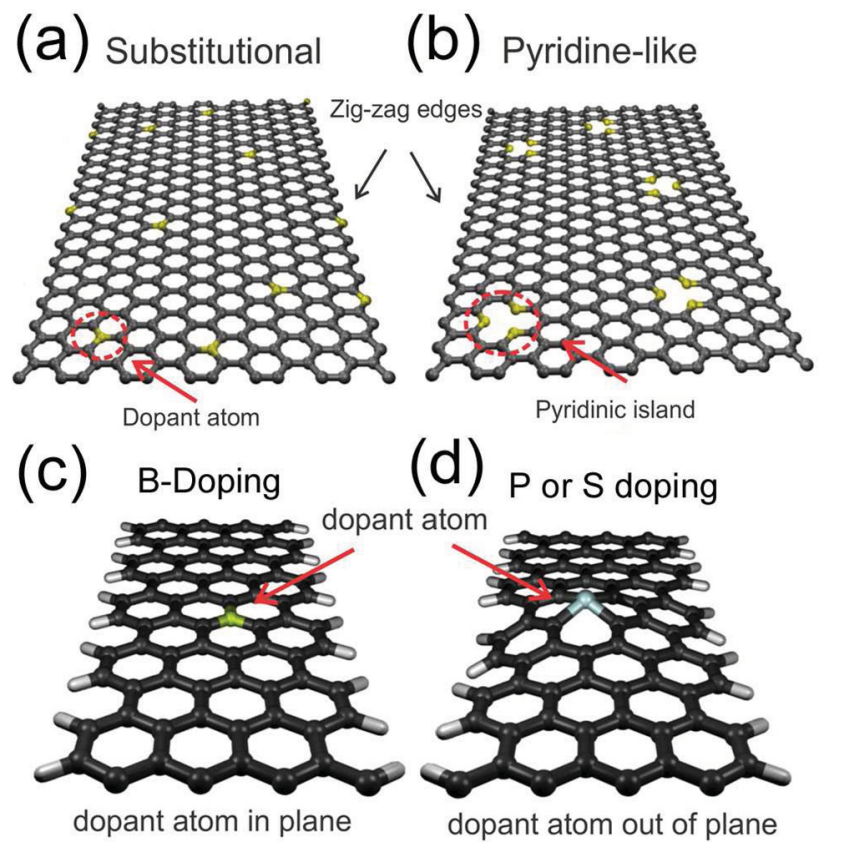

Figure 5. Molecular models representing of possible ways of doping GNRs: (a) Substitutional N-doping; (b) Pyridine-like N-doping, in which a carbon is removed or one vacancy is introduced and the dopant atom replaces the double-coordinated atoms surrounding the vacancy; (c) substitutional B-doping in which the graphene surface does not get distorted by the incorporation of $B$, and (d) substitutional with sulfur (a similar structure also applies for phosphorus); the dopant atom is displaced outside of the plane due to its larger size when compared to carbon (Courtesy of E. Gracia-Espino)

doping could induce a half-metallic behavior. ${ }^{[40]}$ The electronic transport of GNRs doped with different concentration of dopants, has been studied by Roche; ${ }^{[41]}$ different band gaps could be obtained in this way. Although, significant effort has been concentrated on the electronic properties of doped GNRs, further theoretical studies related to the reactivity of dopants and their interactions with different polymer molecules need to be addressed from the theoretical and experimental standpoint.

\section{Key Aspects in Polymer Nanocomposites}

The nanofiller aspect ratio, the percolation threshold and the nature of the nanofiller/matrix interface are key aspects in the polymer nanocomposites design and behavior.

The conceptual framework for understanding mechanical properties of polymer nanocomposites is based on the theory of short fiber composites developed in the second half of the twentieth century. Detailed information can be found elsewhere. ${ }^{[42-44]}$ In this approach, the nanocomposite is considered as a short fiber reinforced polymer with aligned fibers and perfect interfaces except at the fiber ends, where fiber-matrix bonding is disregarded; in this configuration the aspect ratio (AR) is the main parameter affecting composite stiffness (length over diameter for rods and thickness over radius for platelets). Reinforcing potential of aligned 1D nanoparticles (nanotubes) or 2D platelets is typically estimated using simple equations such as the
Halpin-Tsai. ${ }^{[45]}$ For AR values of 1000, simple predictions state that Young's modulus of an ideal (no defects) epoxy composite increases by a factor 6 at $0.01 \%$ filler volume fraction. Nevertheless, using more refined simulations, ${ }^{[46]}$ similar reinforcing potentials are predicted for smaller AR, around 100, which are commonly found in commercial particles.

When the composite is stressed parallel to the fiber axis, analysis of the stress distribution (shear lag analysis, ${ }^{[42-44]}$ ) along the reinforcement length shows that the stress on the fiber is not uniform: the ends of the fibers carry almost no load and the interfacial shear stress is maximum at the end of the fiber. These theoretical results have important consequences in the nanocomposite mechanical behavior. For the matrix to efficiently transfer loads to the fibers, i.e. to transfer the maximum possible load $\left(\mathrm{E}_{\mathrm{f}} \cdot \varepsilon_{\mathrm{m}}\right.$, where $\mathrm{E}_{\mathrm{f}}$ is the Young's modulus of the reinforcement and $\varepsilon_{\mathrm{m}}$ is the matrix strain), these must have a minimum length (alternatively, a minimum AR). Shear lag analysis, although not accurate, allows estimating the minimum AR for 1 TPa nano-reinforcements, yielding values ca. 300 and 1000 for rigid and compliant matrices respectively (one order of magnitude higher than for conventional carbon fibers because these have lower Young's modulus). Although more realistic calculations probably yield lower AR values, extreme care should be taken when preparing nanocomposites to avoid fragmentation of the nano-reinforcement.

The physico-chemical properties of the interfacial regions within nanocomposites need further investigations. Geometrical considerations restrict the interfacial characteristic length to the radius of gyration of typical flexible polymers. Recent experiments using molecular fluorescent probes located at different distances from the filler revealed an interaction distance of $c a .6 \mathrm{~nm}$ in $\mathrm{PMMA} / \mathrm{SiO}_{2}$ nanocomposites, which is defined as the maximum distance from the filler at which the glass transition temperature is different from the bulk polymer matrix. ${ }^{[47]}$ To the best of the authors knowledge, similar studies have not yet been reported for GNRs and GNPs composites, and further research is needed in this direction.

Regarding the interfacial shear stress, if the interfacial region is stronger than the matrix, the matrix will yield; but if weaker, de-bonding may initiate at the fiber end, thus propagating along the interface. This fact justifies the need to engineer the surface of GNRs and GNPs so that the surfaces of the materials strongly interact with different polymer matrices. In general, different types of interactions could occur between a polymer and GNRs: 1) covalent functionalization; 2) non-covalent functionalization and 3) polymer blending (Figure 2). These interactions will depend on the reactivity of the different surface sites within GNRs. For example, covalent functionalization would occur between the edges and the polymer molecules, whereas non covalent interactions take place between the basal plane of GNRs and the polymers. Blending consists on weak interactions of the polymer matrix and the graphene material. Although these interactions may operate at very low strains, the lack of chemical reactivity at the surface of GNRs and GNPs opens the question about its suitability as reinforcing materials against the more reactive graphene oxide (GO) or even doped graphenes, and makes especially relevant to develop chemical routes that take advantage of their reactive edges. But even in the case of no chemical bonding with the polymer matrix, some 
capacity for stress transfer of frictional nature will remain, thus increasing the mechanical damping as Ajayan and co-workers realized for nanotube composites. ${ }^{[48]}$

Transport properties - thermal and electrical - are also strongly modified by the addition of minute amounts of conductive nanofillers and are not linear with the nanoparticle loading. These properties are related to the long-range connectivity of conductive nanofillers. Below a certain loading, nanofillers are not connected and the nanocomposite is essentially non-conductive, and above a certain loading, called percolation threshold or critical fractional volume, conductivity increases abruptly (Figure 6). This value strongly depends on the AR of the conductive nanofillers. The early Coniglio theory of percolation of long rigid rods, ${ }^{[49]}$ predicts that the density of particles scales with the inverse square of the rod aspect ratio. Monte Carlo simulations on randomly oriented uniform rigid nanorods, performed by Foygel, ${ }^{[50]}$ have demonstrated that the critical volume fraction on the verge of the percolation threshold, $\varphi_{c}$, is inversely proportional to AR, being as low as $10^{-3}$ for AR values of about $10^{3}$; polydispersity on nanorod length has the counter-intuitive effect of decreasing $\varphi_{c}$, whereas nanorod flexibility increases $\varphi_{c}{ }^{[51]}$

\subsection{Properties}

The nanocomposites field has emerged to meet the growing demand of almost any industrial sector to overcome the properties of conventional materials. In order to make isolating materials conducting (e.g. polymers) or to increase the mechanical performance and toughness beyond typical polymer thermosets,

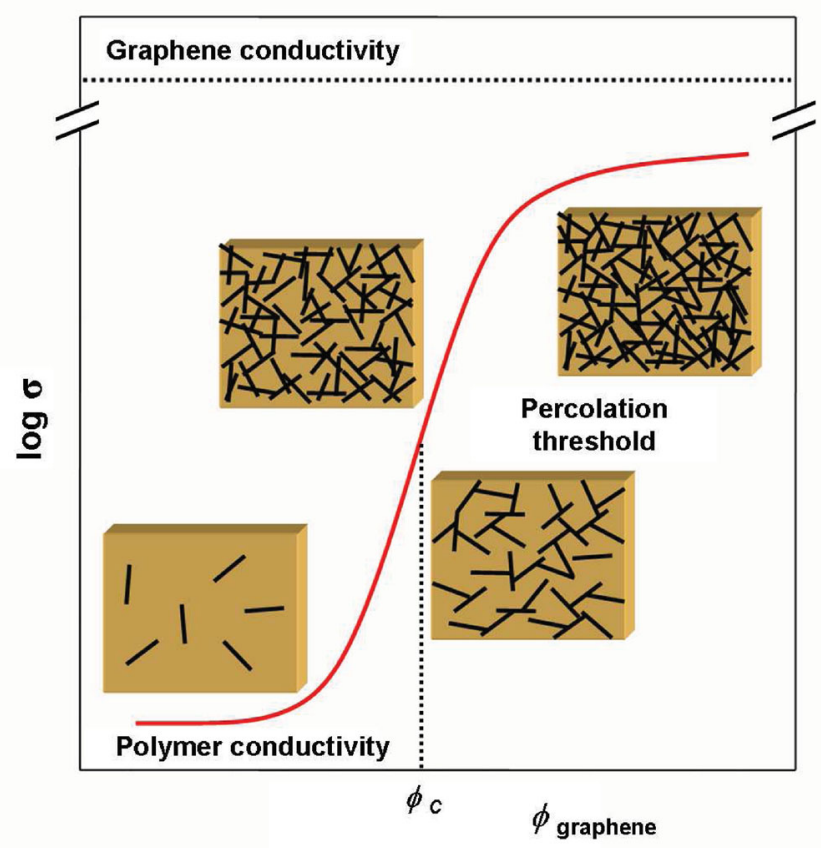

Figure 6. Schematic variation of electric conductivity with filler volume fraction for conductive nanocomposites. $\varphi_{c}$ represents the critical volume fraction at the percolation threshold. it is vital to understand the matrix behavior. For example, in some critical sectors such as aerospace industry there is a pressing need to increase strength and damage resistance in a reliable way, as well as to impart very high electrical conductivity to carbon fiber composites. Its success is of prime importance in order to decrease weight and fuel consumption in aircrafts. In the following sections we describe an updated view of the most relevant properties achieved when some forms of graphene are incorporated into polymers.

\subsection{Mechanical Properties}

The mechanical properties of polymer/graphene nanocomposites (PGN) depend on the dispersion of isolated graphene layers within the polymer matrix and the interfacial bonding established between the nanofiller and the matrix. Although pure graphene is not compatible with polymer matrices and tends to aggregate (layer by layer stacking) due to van der Waals interactions, graphene oxide (GO), which contains hydroxyl and epoxy groups on the sheet and carbonyl and carboxyl groups at the edges, interacts more strongly with polymers. GO is more soluble in water and polar solvents and its surface is relatively easy to modify via the insertion of amines, esters, aromatics or isocyanate functionalities that stabilize dispersions, thus facilitating composite processing. However, pure GO is an insulator and not an optimum filler for fabricating electrically conducting composites. Therefore, an alternative strategy consists of the surface modification of GO for obtaining a good dispersion at a molecular level, and a subsequent reduction to recover, at least partially, the electrical and thermal conductivity via restoring the graphitic network of $\mathrm{sp}^{2}$ hybridized carbon. ${ }^{[52]}$

Although PGN show enhanced stress-strain behavior when compared to conventional composites, we are still far from the values predicted theoretically. For example, one of the maximum increases in Young's modulus found in the literature is reported by Lee: ${ }^{[53]} 200 \%$ increase in Young's modulus for thermoplastic polyurethanes modified with $5 \%$ of graphene. As reported in a recent review, ${ }^{[54]}$ improvements of Young's modulus and tensile strength frequently range between 15-90\% and 60-100\% respectively; elongation at break usually decreases since high $A R$ values and strong interactions with the matrix restrict the mobility of the polymer chains.

The effect of the nanofiller on the matrix properties needs to be more deeply studied since some matrix processes such as phase separation or crystallization may be altered. For example, a significant increment in modulus accompanied by the creation of a polymer crystalline layer on the surface of the nanofiller has been reported for polycaprolactone reinforced with GO nanoplatelets. ${ }^{[55]}$ In contrast $\mathrm{Xu}$ et al. ${ }^{[56]}$ grafted nylon 6 on graphene sheets and observed that the crystallization of chains was depressed. When using graphite nanoplatelets, incomplete exfoliation or restacking of the sheets reduces the AR as well as the mechanical properties of the material. Furthermore, once dispersed, the graphene sheets can adopt distorted structures that reduce the value of the modulus, as they do not have tendency to stretch under tensile stress. The reader is referred to reviews recently published for more detailed information regarding these subjects. ${ }^{[54,57]}$ 
There are basically three methods for fabricating PGNs. The first one is polymerization of the monomer in the presence of the filler, resulting in composites with good mechanical properties and low percolation thresholds (between 0.3 and $1.6 \%$ vol). For example, Nutt and co-workers ${ }^{[58]}$ reported an improvement of $70 \%$ in the tensile strength, and $57 \%$ in the Young's modulus for polystyrene composites, in which the graphene sheets were grafted with polystyrene chains by atomic transfer radical polymerization (ATRP).

The second method is solution compounding. The filler is dispersed in a solution of the polymer, which is then removed after mixing. This method was used to prepare poly(ethyleneco-vinyl acetate) reinforced with expanded graphite and a $100 \%$ increase in modulus was observed with $4 \%$ loading. ${ }^{[59]}$ It has also been used to prepare poly(methylmethacrylate) composites with functionalized graphene sheets (FGS, obtained by thermal expansion of completely oxidized graphite oxide). Here, increments in the Young's modulus and ultimate strengths of $80 \%$ and $20 \%$ respectively, were reported at $0.1 \% \mathrm{w} / \mathrm{w}$ loading. ${ }^{[60]}$ This result demonstrated the ability of FGS to interact with polar polymers for creating a percolated interphase in which the properties of the host polymer matrix were substantially altered.

The last method is melt blending, the best choice from an industrial point of view. Mixing equipments include tworoll mills, extruders and high shear mixers. When using this method, percolation thresholds are usually higher. Commonly, a combination of methods needs to be used. For example, for epoxy nanocomposites, FGS are first dispersed in the monomer with help of a solvent, and high shear mixing and polymerization is performed after solvent removal. ${ }^{[61]}$

The comparison among the mechanical properties obtained from these three main methods or a combination of them, is difficult because of the differences between the systems studied. There are various factors that affect the dispersion degree of the nanofiller and, consequently, the final properties of the material. But there is experimental evidence pointing out that the chemical linkage of the graphene to the polymer matrix enhances the properties of the composite material. The work of Satti et al. ${ }^{[62]}$ on GO/poly(allylamine) is a good example. Here, GO platelets were covalently bonded to the polymer matrix (amide formation between graphene carboxylic groups and polymer matrix amino groups), and theYoung's modulus and the tensile strength increased ca. 200\% and 400\%, respectively.

The performance of PGNs has been much less studied and additional research is needed. In this context, with only $0.125 \%$ $\mathrm{w} / \mathrm{w}$ of FGS, it was observed a $65 \%$ increase in fracture toughness and more than $100 \%$ increase in fracture energy in epoxy/ FGS nanocomposites; ${ }^{[63]}$ a decrease in the fatigue crack growth rate was also reported. Crack deflection seems to be the key in order to understand these outstanding values, as it should be expected for plates with a high AR, unlike nanorods or nanospheres in which other mechanisms as crack bridging are more important. However, some aspects still remain unsolved and further investigations need to be carried out. For example, it has not been clearly established the influence of wrinkles and deformations on the nanosheet, the size and functionalization of the nanoplatelets or the interactions with the polymer matrix on the nanocomposite toughness, or even the matrix transformations induced by graphene. For example, the increment in the gamma phase of nylon 12 loaded with $0.6 \%$ of FGS, increased toughness by $72 \%$ and impact failure by $175 \% .{ }^{[64]}$ Interestingly, PGNs show major increments in fracture toughness and fracture energy than carbon nanotube nanocomposites, ${ }^{[65]}$ and the reasons for observing this behavior should be carefully investigated. ${ }^{[65]}$

\subsection{Electrical Properties}

As shown in Figure 6 , the electrical conductivity, $\sigma_{c}$, of nanocomposites using conductive fillers increases when raising the particle volume fraction. In the vicinity of the percolation threshold, $\sigma_{c}$ can be described using the following equation, ${ }^{[66]}$ in terms of the volume fraction of the filler, $\varphi$ :

$\sigma_{C}=\sigma_{0}\left(\phi-\phi_{c}\right)^{t}$

$\sigma_{0}$ is a pre-exponential factor that depends on the conductivity of the particles, the contacts between them and the topology of the percolation cluster; ${ }^{[50]} \varphi_{c}$ is the fractional volume at the percolation threshold, and $t$ is a universal critical exponent. According Celzard, ${ }^{[67]} t \approx 2.0$, but experimentally, values range between 2 and 6 , depending on the polymeric media. For nanotubes, Foygel, ${ }^{[50]}$ has found that the exponent $t$ is not universal since it depends on AR. In fact, $t=2$ is the limiting value for spherical 3D objects and decreases to 1.2-1.6 for oblong objects with AR $10^{2}-10^{3}$. Hicks, ${ }^{[68]}$ simulated the electrical conductivity of graphene nanocomposites using two values for ARs: width over length and width (or length) over thickness. In this study, it was also found that independently of the filler dimensions (width or thickness), the critical values and exponents strongly depend on the AR.

The intrinsic conductivity of graphene depends on both, the synthesis method and on the surface modification. In particular, the intrinsic conductivity of graphene depends on the number of defects (reactive sites) that can be found on the surface. These defects are usually generated during the oxidation-reduction process of GO. ${ }^{[52,71,72]}$ These processes yield reduced graphenes (RG) with variable carbon-oxygen ratios generating discontinuities on the electronic de-localization of filler and consequently, reducing its conductivity one or two orders of magnitude. Reduction favours sheet crystallization and the formation of graphite. In order to avoid re-stacking of the layers, grafting molecules such as hexylamine, ${ }^{[72]}$ or phenyl isocyanate. ${ }^{[73,74]}$ on the surface are commonly used. However, this chemical grafting generates surface defects on the sheets that reduce their conductivity.

At very low filler loading, the electrical properties are dominated by the dielectric matrix so the composite is essentially non-conductive (Figure 6). As graphene content increases, the dielectric distance that separates the sheets decreases and fillers begin to form clusters in which some graphene-graphene contacts may exist. At the percolation threshold, conduction paths are created all over the volume of the composite and a sudden increase in conduction occurs. Nevertheless, sheet-sheet junctions are highly resistive (for nanotubes, the contact resistance is in the order of $1 \mathrm{M} \Omega$ ), ${ }^{[50]}$ and it has been proposed that the conduction mechanism is probably of the tunnel type. ${ }^{[68]}$ 
Some authors, ${ }^{[50]}$ prevent the use of experimental data at low loadings to estimate the conductivity of the individual particles. But others, ${ }^{[73]}$ used a modified form of equation 1 $\left(\sigma_{C}=\sigma_{f}\left[\left(\phi-\phi_{c}\right) /(1-\phi)\right]^{t}\right)$, and obtained the conductivity of the graphene sheets $\left(10^{4.92} \mathrm{~S} \cdot \mathrm{m}^{-1}\right)$ together with $t(2.74)$ and $\varphi_{c}$ (0.1) in polystyrene nanocomposites.

Current research on graphene surface engineering able to prepare highly conductive materials focuses on the following challenges: a) generate the lowest number of defects within the graphene sheets, b) improve the graphene compatibility with polymer matrices (good dispersion and low percolation threshold), c) impart resistance to the chemical or electrochemical reduction, and c) reduce the sheet-sheet junction resistivity in order to allow electronic transport.

\subsection{Thermal Properties and Relaxations}

Thermal behavior is also deeply modified in PGNs. Graphene significantly increases the thermal stability, especially if the interaction between the matrix and GNPs is strong. For example Liu and co-workers ${ }^{[75]}$ reported a delay in the main stage of degradation at $50{ }^{\circ} \mathrm{C}$ in PS/GNP composites. Moreover, it could significantly increase the thermal conductivity and decrease the thermal expansion coefficient. In this context, Haddon and coworkers, ${ }^{[76]}$ reported that the thermal conductivity of an epoxy resin increased about $400 \%$ with the addition of $5 \%$ of GO. But probably, the main effect is associated to a deep perturbation of the local and global polymer matrix dynamics, thus resulting in changes related to the physical aging as well as the glass transition temperature, ${ }^{[77]} \mathrm{Tg}$ (one of the most important properties of polymers). The behavior near $T_{\mathrm{g}}$ is critical for high-temperature applications and for polymer composite processing.

To date the restricted relaxation behavior of nanocomposites in the presence of graphene sheets requires further exploration and investigation. Only few articles report $T_{g}$ values of PGNs. It has been recently observed, ${ }^{[60]}$ an spectacular increase in $T_{\mathrm{g}}$ of $30{ }^{\circ} \mathrm{C}$ in PMMA with only $0.05 \mathrm{wt} \% \mathrm{FGS}$, and of $46{ }^{\circ} \mathrm{C}$ in poly(acrylonitrile) loaded with $1 \%$ wt FGS. These results were attributed to: a) the FGS wrinkled morphology that enhances mechanical interlocking with the polymeric chain and, therefore, a better interfacial adhesion, and b) the presence of oxygenated groups on graphene surface which may form hydrogen bonds with the carbonyl groups of acrylic polymers such as PMMA. The effect of covalent bonding of poly(vinyl chloride) to reduce $\mathrm{GO}$ has also been studied, ${ }^{[78]}$ and a significant increase in $T_{\mathrm{g}}$ of $25{ }^{\circ} \mathrm{C}$ for $1.4 \%$ wt loading, along with an increase of $50 \%$ in the Young's modulus, have been found.

The structural interpretation of the shifts in $T_{\mathrm{g}}$ with increasing nanoparticle loading often does not have suitable support in the current physics of these nanomaterial systems. The volume of the rigid filler, local curvature, polymer stiffness and interfacial interactions seem to be important contributions to interpret $T_{\mathrm{g}}$ variations. Jancar et al. ${ }^{[79]}$ have reviewed the physical origin of changes in $T_{\mathrm{g}}$ (or chain relaxation behavior) showing that: a) attractive surface interactions lead to a slowing of dynamics near the surface, resulting in an increase of viscosity which grows linearly with nanoparticle concentration if clustering is avoided, b) high aspect ratios or highly asymmetric nanoparticles are more effective in changing the properties of the polymer matrix because they could more easily form entanglements and network structures (for example through chain bridging between the nanoparticles), and c) the grafting of polymer brushes and the strength of their interaction with the polymer matrix determines the macroscale properties.

\subsection{Optical Properties}

The absorption spectrum of graphene monolayers is flat and monotonous in the wavelength range between 400 and $750 \mathrm{~nm}$. In this range, it absorbs a small fraction of white light, ${ }^{[80]}(2.3 \%)$, which increases with the number of layers, and its reflectance is negligible $(<0.1 \%)$. Pure graphene may be useless as filter or absorbing material in the visible range, but it remains as the most transparent conductive material that may be used to fabricate electrodes for photovoltaic devices, ${ }^{[81]}$ or may give rise to novel phenomena by experiencing subtle physicochemical interfacial modifications.

For example, An and co-workers ${ }^{[82]}$ covered graphene flakes obtained by drying a graphene suspension, with 1-pyrenecarboxylic acid (PCA), a pericondensed aromatic molecule which absorbs light around $340 \mathrm{~nm}$, and forms non covalent $\pi-\pi$ binding sites with graphene layers. The authors were able to make a flexible photosensitive device by embedding the graphene/PCA ensemble in a silicone matrix. Opposite to the majority of photoconductors, the resistance increased in the presence of white light; the mechanism is not clear yet but diffusion of oxygen triggered by light should not be excluded as a possible mechanism.

GO acts as a strong quencher for several luminescent dyes. $\mathrm{Xu}$ et al. ${ }^{[83]}$ grafted a porphyrin (5-4(aminophenyl)-10,15,20triphenyl porphyrin, TPP) to GO via amide bonding, and found that luminescence from TPP was strongly quenched. This was attributed to two possible competitive processes: photo-induced electron transfer and intra-molecular energy transfer facilitated by a through-bond mechanism due to the covalent binding of the dye. It was also found that the donor-acceptor hybrids exhibited a superior optical limiting performance, i.e., they exhibited high transmittance of low-intensity light, and attenuated intense optical beams.

Quenching could also occur in the absence of covalent binding. Kozehemyakina and co-workers, ${ }^{[84]}$ attributed the quenching of perylene bismide fluorescence by the presence of graphene in the solid state to a photo-induced electron transfer mechanism. The empty states in the conduction band above the Fermi level in graphene could act as acceptor for the photoexcited electron of the dye, whereas the filled valence band of graphene could act as a donor for electrons able to refill the remaining holes in the ground state of the dye. This ability of graphene to quench fluorescence emission has been successfully applied by Kim and co-workers, ${ }^{[85]}$ to obtain images of graphene layers with a simple fluorescence microscope.

\subsection{Barrier Properties}

It is well known that permeability of composites depend on the solubility of the gas (both in the matrix and the reinforcement), 
and its diffusivity. Diffusion of a gas through a composite is intimately related with the tortuosity of the path, and this is the main reason why particles with high AR enhance the barrier properties. Consequently, exfoliated nanoclays have been extensively used for this purpose.

Nano graphenes could have a very high aspect ratio and therefore very low solubility to gases due to the size and high electronic density of the carbon rings, able to repel atoms and gas molecules. It has been reported that defect free graphene is impermeable even to Helium. ${ }^{[86]}$ These features make graphene an excellent candidate for enhancing barrier properties of polymers. During the last years, a few research papers related to the barrier properties of pristine graphene and PGNs have been reported, revealing enhanced barrier properties at lower loadings when compared to nano-clay composites. ${ }^{[87,88]}$

Nanocomposites of crumpled GO functionalized with phenyl isocyanate in a polystyrene (PS) matrix, ${ }^{[87]}$ exhibiting a high number of $\pi-\pi$ interfacial interactions, showed exceptionally low permeability to oxygen at very low loading (0.02\%) (similar permeability was obtained with nanoclays but at $1-1.5 \%$ loading). The AR and filler alignment also influence the gas permeation through a polymer composite. For instance, when the filler is misaligned the barrier properties to $\mathrm{He}$ and $\mathrm{N}_{2}$ in polycarbonate PGN are not as good as those observed for well aligned nanocomposites. ${ }^{[88]}$ Graphene also enhances the barrier properties of LDPE towards organic solvents such as toluene, ${ }^{[89]}$ although in this case the improvement has not been as large (56\% to 39\%) as for the cases mentioned above.

Defects are also important for barrier properties, and we should not forget that the atomic perforation of the graphene layer may be generated as a consequence of the manufacture process. ${ }^{[89]}$ Nevertheless, the possibility of having sub-nanometric holes opens a wide field for the use of graphenes with tailored functionalized pores as gas separation membranes. Molecular dynamics simulations have shown that hydrogen and methane could be effectively separated using a graphene sheet. ${ }^{[90]}$

\section{Conclusions}

In the future, alternative methods able to produce bulk amounts of graphene, graphene nanoribbons, doped nanoribbons and graphene platelets need to be explored. It is vital to control the type and amount of reactive sites on the graphene surface and edges as well as to discover procedures for massive production of graphene at a lower cost. It seems that covalent bonds need to be established between the polymer matrix and the graphene filler in order to fabricate robust nanocomposites with extraordinary mechanical behavior and gas barrier properties but at the expense of electrical conductivity; methods to decrease sheet-sheet resistance, perhaps via graphene welding, are needed. Partially reduced GO seems to be a way to fabricate polymer composites, however, the amount of oxygen and the degree of crystallinity needs further control. The use of doped graphenes has not been reported hitherto, and it is believed that these doped materials could result in high performance nanocomposites. Conducting and transparent polymer composites using graphenes or doped graphenes need further investigations; it may be that graphene composites could replace indium tin oxide (ITO) but enhanced electrical conductivities of the fillers need to be increased by orders of magnitude. The area of graphene nanocomposites is emerging and this appears to be the tip of the iceberg, since a vast number of composites for different applications still need to be synthesized, studied and optimized.

Authors wish to thank Ministerio de Ciencia e Innovación (SPAIN) for financial support under grant MAT2010-17091. MT and SMVD acknowledge support from the Research Center for Exotic NanoCarbon Project, Japan regional Innovation Strategy Program by the Excellence, JST.

\section{References}

[1] H. P. Boehm, A. Clauss, G. Fischer, U. Hofmann, Proc. of the Fifth Conference on Carbon.Pergamon Press, London, 1962, pp.73.

[2] a) K. S. Novoselov, A. K. Geim, S. V. Morozov, D. Jiang, Y. Zhang, S. V. Dubonos, I. V. Grigorieva, A. A. Firsov, Science 2004, 306, 666 ;b) K.S. Novoselov, A. K. Geim, S.V. Morozov, D. Jiang, M.I. Katsnelson, I.V. Grigorieva,S.V.Dubonos,A.A.Firsov,Nature 2005,438,197;c)Y.B.Zhang,Y.W.Tan,H. L.Stormer,P.Kim, Nature2005,438,201.

[3] K. S. Novoselov, Z. Jiang, Y. Zhang, S. V. Morozov, H. L. Stormer, U. Zeitler, J. C. Maan, G. S. Boebinger, P. Kim, A. K. Geim, Science 2007, 315, 1379.

[4] C. Lee, X. D. Wei, J. W. Kysar, J. Hone, Science 2008, 321, 385

[5] A. K. Geim, K. S. Novoselov, Nat. Mater. 2007, 6, 183.

[6] Y. Zhang, T. Tang, C. Girit, Z. Hao, M.C. Martin, A. Zettl, M. F. Crommie, Y. R. Shen, F. Wang, Nature 2009, 459, 820.

[7 S. V. Morozov, K. S. Novoselov, F. Schedin, D. Jiang, A. A. Firsov, A. K. Geim, Phys. Rev. B 2005, 72, 201401.

[8] A. A. Balandin, S. Ghosh, W. Bao, I. Calizo, D. Teweldebrhan, F. Miao, C. N. Lau, Nano Lett. 2008, 8, 902.

[9] W. Cai, A. L. Moore, Y. Zhu, X. Li, S. Chen, L. Shi, R. S. Ruoff, Nano Lett. 2010, 10, 1645.

[10] I. W. Frank, D. M. Tanenbaum, A. M. van der Zande, P. L. McEuen, J. Vac. Sci. Technol. B 2007, 25, 2558.

[11] K. S. Kim, Y. Zhao, H. Jang, S. Y. Lee, J. M. Kim, K. S. Kim, J. Ahn, P. Kim, J. Choi, B.H. Hong, Nature 2009, 457, 706.

[12] S. B. Bon, L. Valentini, R. Verdejo, J. L. Garcia Fierro, L. Peponi, M. A. Lopez-Manchado, J. M. Kenny, Chem. Mater. 2009, 21, 3433.

[13] S. Osuna, M. Torrent-Sucarrat, M. Solà, P. Geerlings, C. P. Ewels, G. V. Lier, J. Phys. Chem. C 2010, 114, 3340.

[14] B. Wang, B. J. Cooley, S. Cheng, K. Zou, Q. Z. Hao, F. Okino, J. Sofo, N. Samarth, J. Zhu, in APS March Meeting 2010 Abstracts, American Physical Society, 2010.

[15] J. O. Sofo, A. S. Chaudhari, G. D. Barber, Phys. Rev. B 2007, 75, 153401[16] A. K. Geim, K. S. Novoselov, D. C. Elias, R. R. Nair,T. M. G. Mohiuddin,

S. V. Morozov, P. Blake, M. P. Halsall, A. C. Ferrari, D. W. Boukhvalov,M. I. Katsnelson, Science 2009, 323, 610.

[1] D. A. Dikin, S. Stankovich, E. J.Zimney, R. D. Piner, G. H. B. Dommett, G. Evmenenko, S. T. Nguyen, R. S. Ruoff, Nature 2007, 448, 457.

[18] S. Park, R.S. Ruoff, Nat. Nanotechnol. 2009, 4, 217.

[19] C. Berger, Z. Song, X. Li, X. Wu, N. Brown, C. Naud, D. Mayou,

T. Li, J. Hass, A. N. Marchenkov, E. H. Conrad, P. H. First, W. A. Heer, Science 2006, 312, 1191.

[20] A. Reina,X.Jia, J. Ho, D. Nezich, H.Son, V. Bulovic, M. S. Dresselhaus,

J. Kong, Nano Lett. 2009, 9, 30. 
[21] S. Zhengzong, Z. Yan, J. Yao, E. Beitler, Y. Zhu, J. M. Tour, Nature 2010, 468, 549.

[22] X. Li, W. Cai, J. An, S. Kim, J. Nah, D. Yang, R. Piner, A. Velamakanni, I. Jung, E. Tutuc, S. K. Banerjee, L. Colombo, R. S. Ruoff. Science 2009, 324, 1312.

[23] J. Campos-Delgado, J. M. Romo-Herrera, X. T. Jia, D. A. Cullen, H.Muramatsu,Y.A.Kim,T.Hayashi,Z.F.Ren,D.J.Smith,Y.Okuno,T.Ohba,H.Kanoh,K. Kaneko,M.Endo,H.Terrones,M.S.Dresselhaus,M.Terrones,Nano Lett.2008,8,2773.

[24] H. Murayama, T. Maeda, Nature 1990, 345, 791.

[25] X. Li, X. Wang, L. Zhang, S. Lee, H. Dai, Science 2008, 319, 1229.

[26] D. Wei, Y. Liu, H. Zhang, L. Huang, B. Wu, J. Chen, G. Yu, J. Am.

Chem. Soc. 2009, 131, 11147.

[27] X. Yang, X. Dou, A. Rouhanipour, L. Zhi, H. J. Rader, K. Mullen, J. Am. Chem. Soc. 2008, 130, 4216.

[28] Y. Xiong, Y. Xie, X. Li, Z. Li, Carbon 2004, 42, 1447.

[29] Z. Kang, E. Wang, B. Mao, Z. Su, L. Gao, S. Lian, L. Xu, J. Am. Chem. Soc. $2005,127,6534$

[30] A. M. Affoune, B. L. V. Prasad, H. Sato, T. Enoki, Y. Kaburagi, Y. Hishiyama, Chem. Phys. Lett. 2001, 348, 17

[31] A. G. Cano-Márquez, F. J. Rodríguez-Maclas, J. Campos-Delgado, C.G.Espinosa-Gonzaález,F.Tristán-López,D.Ramírez-González,D.A.Cullen,D.J. Smith,M.Terrones,Y.I.Vega-Cantú,Nano Lett. 2009,9,1527.

[32] L. Jiao, L. Zhang, X. Wang, G. Diankov, H. Dai, Nature 2009, 458, 877.

[33] D. V. Kosynkin, A. L. Higginbotham, A. Sinitskii, J. R. Lomeda, A. Dimiev, B. K. Price, J. M. Tour, Nature 2009, 458, 872.

[34] M. Terrones, Nature 2009, 458, 845.

[35] A. L. Elías, A. R. Botello-Méndez, D. Meneses-Rodríguez, V. J. González, D. Ramírez-González, L. Ci, E. Muñoz-Sandoval P. M. Ajayan, H. Terrones, M. Terrones, Nano Lett. 2010, 10, 366[36] K. Kim, A. Sussman, A. Zettl, ACS Nano 2010, 4, 13626.

[37] M.Terrones,A.R.Botello-Méndez,J.Campos-Delgado,F.López-Urlas,

Y.I.Vega-Cantú,F.J.Rodríguez-Macías,A. L.Elias, E.Muñoz-Sandoval,A. G. CanoMárquez, J.-C. Charlier, H. Terrones, Nano Today 2010, 5, 351.

[38] X. Jia, M. Hofmann, V. Meunier, B. G. Sumpter, J. Campos-Delgado,

J. M. Romo-Herrera, H. Son, Y. Hsieh, A. Reina, J. Kong

M. Terrones, M. S. Dresselhaus, Science 2009, 323, 17015.

[39] F. Cervantes-Sodi, G. Csanyi, S. Piscanec, A. C. Ferrari, Phys. Rev. B 2008, 7765427.

[40] S. Yu, W. Zheng, Q. Wen, Q. Jiang, Carbon 2008, 46, 537.

[41] B. Biel B, F. Triozon, X. Blase, S. Roche, Nano Lett. 2009, 9, 2725[42] D. Hull, in An introduction to composite materials, Cambridge University Press, Cambridge 1996, Chap. 6.

[43] J.-K. Kim, Y.-W. Mai in Structure and Properties of Composites, Vol 13 (Ed: T. W. Chou), VCH, Weinheim 1993, Ch 6.

[44] M. G. Bader, A. R. Hill, in Structure and Properties of Composites, Vol 13 (Ed: T. W. Chou), VCH, Weinheim 1993, Ch 7.

[45] J. C. Halpin, J. L. Kardos, Polym. Eng. Sci. 1976, 16, 344.

[46] A. A. Gusev, Macromolecules 2001, 34, 3081

[47] K. Parker, R. T. Schneider, R. W. Siegel, R. Ozisik, J. C. Cabanelas,

B. Serrano, C. Antonelli, J. Baselga, Polymer 2010, 51, 4891.

[48] P. M. Ajayan, J. M. Tour, Nature 2007, 447, 1066.

[49] A. Coniglio, U. de Angelis, A. Forlani, J. Phys. A 1977, 10, 1123.

[50] M. Foygel, R. D. Morris, D. Anez, S. French, V. L. Sobolev, Phys. Rev. B 2005, 71, 104201.

[51] T. Schilling, S. Jungblut, M. A. Miller, in Handbook of Nanophysics: Nanotubes and Nanowires, (Ed: K. D. Sattler), CRC Press, Taylor \&Francis, Boca Ratón, US, 2011, Ch 23.

[52] I. K. Moon, J. Lee, R. S. Ruoff, H. Lee, Nat. Commun. 2010, 1, 1.

[53] Y. R. Lee, A. V. Raghu, H. M. Jeong, B. K Kim, Macromol. Chem. Phys. 2009, 210, 1247.

[54] T. Kuilla, S. Bhadra, D. Yao, N.H. Kim, S. Bose, J.H. Lee, Prog. Polym. Sci. 2010, 3, 1350.
[55] D. Cai, M. Song, Nanotechnology 2009, 20, 315708.

[56] Z. Xu, C. Gao, Macromolecules 2010, 43, 6716.

[57] R. Sengupta, M. Bhattacharya, S. Bandyopadhyay, A.K. Bhowmick,

Prog. Polym. Sci. 2011, 36, 638.

[58] M. Fang, K. Wang, H. Lu, Y. Yang, S. Nutt, J. Mater. Chem. 2009, 19 7098.

[59] J. J. George, A. K. Bhowmick, J. Mater. Sci 2008, 43, 702.

[60] T. Ramanathan, A. A. Abdala, S. Stankovich, D. A. Dikin, M.Herrera-Alonso,R.D.Piner,D.H.Adamson,H.C.Schniepp,X.Chien,R.S.Ruoff,S.T. Nguyen,I.AAksay,R.K.Prud 'Homme,L.C.Brinson,Nat. Nanotechnol.2008,3,327.

[61] J. Liang, Y. Wang, Y. Huang, Y. Ma, Z. Liu, J. Cai, C. Zhang, H. Gao, Y. Chen, Carbon 2009, 47, 922.

[62] A. Satti, P. Larpent, Y. Gun'Ko, Carbon. 2010, 48, 3376.

[63] M.A. Rafiee, J. Rafiee, I. Srivastava, Z. Wang, H. Song, Z.-Z. Yu, N. Koraktar, Small 2010, 6, 179.

[64] R. Rafiq, D. Cai, J. Jin, M. Song, Carbon 2010, 48, 4309.

[65] M.A. Rafiee, J. Rafiee, Z. Wang, H. Song, Z.-Z. Yu, N. Koraktar, ACS Nano 2009, 3, 3884

[66] D. Stauffer, A. Aharnoy, Introduction to Percolation Theory, Taylor \& Francis, London, 1991, Ch. 5.

[67] A. Celzard, J. F. Marêch, Physica A 2003, 317, 305

[68] J. Hicks, A. Behnam, A. Uralb, Appl. Phys. Lett. 2009, 95, 213103.

[69] S. Unarunotai, Y. Murata, C. E. Chialvo, N. Mason, I. Petrov,

R. G. Nuzzo, J. S. Moore, J. A. Rogers, Adv. Mater. 2010, 22, 1072[70] Y. Zhu, S.

Murali, W. Cai, X. Li, J. W. Suk, J. R. Potts, R. S. Ruoff, Adv. Mater. 2010, 22, 3906

[71] H. Kim, A. A. Abdala, C. W. Macosko, Macromolecules 2010, 43, 6515.

[72] O. C. Compton, D. A. Dikin, K. W. Putz, L. C. Brinson, S. T. Nguyen, Adv. Mater. 2010, 22, 892.

[73] S. Stankovich, D. A. Dikin, G. H. B. Dommett, K. M. Kohlhaas, E. J. Zimney, E. A. Stach, R. D. Piner, S. T. Nguyen, R. S. Ruoff, Nature 2006, 442, 282.

[74] G. Eda, M. Chhowalla, Nano Lett. 2009, 9, 814.

[75] N. Liu, , H. Wu, Y. Liu, C. Zhang, J. Chen, Adv. Funct. Mat. 2008, 18, 1518.

[76] A. Yu, P. Ramesh, X. Sun, E. Bekyarova, M. E. Itkis, R. C. Haddon, Adv. Mater. 2008, 20, 4740.

[7] A. L. Flory, T. Ramanathan, L. C. Brinson, Macromolecules 201043 4247.

[78] H. J. Salavagione, G. Martínez, Macromolecules 2011, 44, 2685.

[79] J. Jancar, J. F. Douglas, F. W. Starr, S. K. Kumar, P. Cassagnau, A. J. Lesser, S. S. Sternstein, M. J. Buehler, Polymer 2010 51, 3321[80] R. R. Nair, P. Bake, A. N. Grigorenko, K. S. Novosellov, T. J. Booth, A. K. Geim, Science 2008, 320, 1308.

[81] Z. Yin, S. Sun, T. Salim, S. Wu, X. Huang, Q. He, Y. M. Lam, H. Zhang. ACS Nano 2010, 4, 5263.

[82] X. An, T. W. Butler, M. Washington, S. K. Nayak, S. Kar, ACS Nano 2011, 5, 1003.

[83] Y. Xu, Z. Liu, X. Zhang, Y. Wang, J. Tian, Y. Huang, Y. Ma, X. Zhang Y. Chen, Adv. Mater. 2009, 21, 1275.

[84] N. V. Kozhemyakina, J. M. Englert, G. Yang, E. Spiecker,

C. D. Schmidt, F. Hauke, A. Hirsch, Adv. Mater. 2010, 22, 5483 [85] J. Kim, L. J. Cote, F. Kim, J. Huang, J. Am. Chem. Soc. 2010, 132, 260.

[86] J. Bunch, S. S. Verbridge, J. S. Alden, A. M. Van der Zande, J. M. Parpia, H. G. Craighead, P. L. McEuen, Nano Lett. 2008, 8 , 2458.

[87] O. C Compton, S. Kim, C. Pierre, J. M. Torkelson, S. T. Nguyen, Adv. Mater. 2010, 22, 4759.

[88] H. Kim, C.W. Macosko, Polymer 2009, 50, 3797.

[89] J. Wang, C. Xu, H. Hu, L. wan, R. Chen, H. Zheng, F. Liu, M. Zhang X. Sheng, X. Wang, J. Nanopart. Res. 2011, 13, 869.

[90] D. Jiang, V.R. Cooper, S. Dai, Nano Lett. 2009, 9, 4019. 